\title{
AVALIAÇÃO DA QUALIDADE DE VIDA E EQUILÍBRIO EM IDOSAS SEDENTÁRIAS E PRATICANTES DE ATIVIDADE FÍSICA
}

\author{
Mansueto Gomes Neto Fisioterapeuta, mestre em Ciências da \\ reabilitação pela UFMG e professor da \\ União Metropolitana de Educação e \\ Cultura - Lauro de Freitas e \\ Universidade do Estado da Bahia \\ e Universidade Federal da Bahia.
}

\author{
Cristiano Sena da Conceição Fisioterapeuta, mestre em Engenharia e \\ Gestão do Conhecimento pela UFSC e \\ professor da União Metropolitana de \\ Educação e Cultura - Lauro de Freitas. \\ Estácio-FIB e Universidade Federal da \\ Bahia. \\ Paulo Henrique Eufrásio de Oliveira Coordenador do curso de Fisioterapia da \\ União Metropolitana de Educação e \\ Cultura - Lauro de Freitas. \\ Luciano Prado Junior Acadêmico do curso de Fisioterapia pela \\ União Metropolitana de Educação e \\ Cultura - Lauro de Freitas.
}

\begin{abstract}
Resumo
Introdução: Dentre as alterações fisiológicas no idoso, a perda da manutenção do equilíbrio merece maior atenção devido aos altos índices de quedas que são uma das principais causas de morbi-mortalidade nesta faixa etária. Portanto, objetiva-se com o presente estudo verificar se a pratica de atividade física interfere positivamente na condição de equilíbrio e QV em idosas. Métodos: Trata-se de um estudo quantitativo de caráter transversal, analítico com idosas com capacidade de deambular independentemente. As voluntárias foram divididas entre os grupos ativo (GA) e sedentário (GS) e submetidas à avaliação através dos questionários IASF, SF-36 e Berg, seguido da análise descritiva e inferencial através do test $t$ de Student utilizando o software Statistical Package for Social Science (SPSS - versão 17.0). Resultados: A amostra foi composta por 45 idosas divididas em 20 para o GS e 25 para o GA. A média de idade entre os grupos foi de 72,08 \pm 7,12 no GA e 68,95 \pm 9,62 no GS, não apresentando diferença estatisticamente significativa. Houve diferença significativa para o IASF no qual o GA apresentou maior grau de instrução e maior condição financeira comparado ao GS, no questionário Berg o GA apresentou maior escore comparado ao GS, no questionário SF-36 houve maior escore no GA no domínio Capacidade funcional e GS para o domínio Estado geral de saúde. Conclusão: O estudo sugere que idosos praticantes de atividades físicas regulares, apresentam melhor equilíbrio e melhores capacidades funcionais comparados a idosos sedentários, que apresentaram melhor estado geral de saúde em divergência com a literatura.
\end{abstract}

Palavras-chave: Envelhecimento; Sedentarismo; Equilíbrio Postural; Exercício.

\begin{abstract}
Introduction: Among the physiological changes in the elderly, loss of balance maintenance needs greater attention, given the high incidence of falls which are a major cause of morbidity and mortality in this age group. Therefore, the objective is to verify that the current study the practice of physical activity positively affects the equilibrium condition and QOL in elderly women. Methods: This is a quantitative study of transversal and analytical composed of older women, with the ability to walk independently. The volunteers included in the study were divided between the active (GA) and sedentary (GS) were subjected to evaluation through questionnaires IASF, SF-36 and Berg, followed by data analysis by Student $t$ test and analysis of statistical data using the Statistical Package for Social
\end{abstract}


Sciences (SPSS - version 17.0). Results: The sample consisted of 45 elderly divided by 20 to 25 for the GS and GA. The average age among the groups was $72.08 \pm 7.12$ in the GA and $68.95 \pm 9.62$ in the GS, no statistically significant differences. There were significant differences for the IASF in which GA had higher education level and higher financial condition compared to GS, Berg questionnaire in the GA had higher scores compared to GS, the SF-36 score was higher in the GA domain and functional capacity GS domain for the General State of Health. Conclusion: The study suggests that elderly practitioners of regular physical activity have better balance and better functional abilities compared to sedentary elderly people who experienced better overall health status at odds with the literature.

Keywords: Aging; Sedentary Lifestyle; Postural Balance; Exercise.

\section{INTRODUÇÃO}

Entende-se envelhecimento como um processo fisiológico marcado por uma regressão biossocial, que é observada em todos os seres vivos. Neste processo ocorre a perda de capacidade psicofísica ao longo da vida influenciado por diversas variáveis como as genéticas, hábitos de vida, além de alterações fisiológicas inerentes ao envelhecimento celular. ${ }^{(1)}$

A quantidade de idosos vem aumentando consideravelmente no Brasil. Este quadro é atribuído ao aumento da expectativa de vida, a diminuição da taxa de natalidade, a um melhor controle de doenças infecto-contagiosas (imunização) e crônico-degenerativas. ${ }^{(2,3)}$ Evidencia-se com este fato a importância de garantir aos idosos não só uma sobrevida maior, mas também uma boa qualidade de vida. ${ }^{(4)}$

As alterações inerentes ao processo de envelhecimento são destacadas pelas mudanças fisiológicas que implicam na diminuição da capacidade funcional e do desempenho motor para manutenção do equilíbrio corporal e realização das atividades da vida diária (AVD). ${ }^{(5,6)}$

O equilíbrio ou manutenção da estabilidade está relacionado ao balanceamento entre forças internas e externas que agem no corpo durante a realização de ações motoras. ${ }^{(5,6)}$ Desta forma, alterações sensoriais, motoras e posturais decorrentes do envelhecimento, podem prejudicar esta manutenção do equilíbrio, levando a uma predisposição à ocorrência de quedas que representam uma importante causa de morbimortalidade nesta faixa etária mais avançada de vida. $^{(7)}$

As quedas no idoso podem produzir restrições nas atividades da vida diária, incapacidades, declínio na saúde e aumento do risco de institucionalização, propiciando não apenas prejuízo físico, mas também psicológico. ${ }^{(7)}$ Para a OMS, as quedas representam um dos problemas mais importantes e comuns relatados entre os idosos, que aumentam progressivamente a cada década de vida. ${ }^{(8)}$ 
O conceito de Qualidade de Vida $(\mathrm{QV})$ está relacionado à auto-estima e ao bem estar pessoal e abrange um grande número de domínios que são importantes à vida do sujeito, compreendendo um caráter multidimensional dependente da integração da saúde física, bemestar psicológico, satisfação social e pessoal. ${ }^{(9)}$ Com o avanço da idade, as alterações no aparelho locomotor repercutem sobre a funcionalidade do indivíduo, facilitam um processo de fragilidade e dependência, impactando na redução da QV. ${ }^{(10)}$

Neste sentido o envelhecimento associado ao sedentarismo se apresenta como uma das principais causas para as doenças crônicas degenerativas. Por outro lado, a prática de exercícios, além de combatê-lo, contribui de maneira significativa para a manutenção da aptidão física e funcional, reduzindo o risco de quedas em idosos e trazendo melhorias para a QV e no bem estar biopsicossocial. $^{(5,7)}$

Diante do aumento da população idosa mundial e a alta incidência de quedas em idosos, é necessária a produção de evidências que confirmem a importância da prática da atividade

física na terceira idade como um método terapêutico para a manutenção da QV e prevenção de quedas. Assim, o objetivo do presente estudo foi verificar se a atividade física interfere positivamente na condição de equilíbrio e QV em idosas.

\section{METODOLOGIA}

Foi realizado um estudo quantitativo de caráter transversal, analítico e comparativo. Foram incluídas no estudo mulheres acima de 60 anos, residentes na cidade de Salvador com capacidade para deambular independentemente. Foram excluídas idosas incapazes de realizar o protocolo estabelecido pela pesquisa. Antes da realização da pesquisa, as idosas foram esclarecidas sobre os procedimentos e objetivos da pesquisa e assinaram o TCLE, e a pesquisa foi aprovada pelo Comitê de Ética e Pesquisa da Faculdade de Tecnologia e Ciências (FTC), sob o parecer $n^{\circ} 1.872$.

As voluntárias foram divididas em dois grupos, um grupo de idosas fisicamente ativas denominadas GA, composto por idosas praticantes de atividade física, e um grupo de idosas sedentárias, composto por idosas que não praticavam nenhuma atividade física, classificadas como sedentárias e denominadas GS. Para a coleta de dados sócio-demográficos foi aplicado um 
questionário sócio-funcional (IASF), para avaliar o equilíbrio, a escala de equilíbrio de Berg e a QV foi avaliada através do questionário SF-36.

O IASF é um instrumento que foi criado para auxiliar os profissionais de saúde a realizarem diagnósticos da situação de saúde em idosos, que pudesse contribuir na elaboração de projetos terapêuticos individuais, de acordo com as necessidades apresentadas e contemplando as várias dimensões do ser humano em processo de envelhecimento. ${ }^{(12)}$

A escala de Equilíbrio de Berg avalia o equilíbrio do indivíduo em 14 situações, representativas de atividades diárias, tais como: ficar de pé, levantar-se, andar, inclinar-se à frente, transferir-se e virar-se. A pontuação máxima a ser alcançada é de 56 pontos e cada item possui uma escala ordinal de cinco alternativas variando de 0 a 4 pontos, de acordo com o grau de dificuldade. ${ }^{(13)}$

O SF-36 é um instrumento genérico de avaliação de QV, de fácil administração e compreensão. É um questionário multidimensional formado por 36 itens, englobados em 8 escalas ou componentes: capacidade funcional, aspectos físicos, dor, estado geral da saúde, vitalidade, aspectos sociais, aspectos emocionais e saúde mental. Apresenta um escore final de 0 a 100, no qual zero corresponde ao pior estado geral de saúde e 100 ao melhor estado de saúde. ${ }^{(14)}$

Foi realizada uma análise descritiva dos dados obtidos por meio de construção de tabelas e do cálculo de variáveis estatísticas, de forma que os dados de variáveis contínuas foram analisados com medidas de tendência central e dispersão e expressos como médias, medianas e desvio-padrão. Os dados de variáveis dicotômicas ou categóricas foram analisados com medidas de freqüência e expressos como porcentagens.

Para a realização da estatística inferencial foram realizados inicialmente testes para normalidade (teste de Kolmogorov-Smirnov) e de homogeneidade de variância para todas as variáveis. Como os dados se encontraram normalmente distribuídos, o teste $t$ de student para amostras independentes foi utilizado para comparar as diferenças das médias das variáveis entre os grupos, as diferenças entre as mudanças nos valores médios foram expressas com um intervalo de confiança de 95\%. O nível de significância estabelecido foi de 5\%. A análise estatística foi realizada com o uso do software SPSS (Statistical Package for the Social Sciences) for Windows (versão 14.0). 


\section{RESULTADOS}

A amostra da pesquisa foi composta por 45 idosas, divididas em um grupo de 20 idosas sedentárias e outro grupo de 25 idosas fisicamente ativas. Não houve nenhuma desistência das participantes, nem ocorreram intercorrências que as impossibilitassem de permanecer na pesquisa.

A média de idade do GA foi de 72,08 $\pm 7,12$ e do GS apresentou média de idade de $68,95 \pm 9,62$, de forma que esta diferença não foi estatisticamente significativa. Os resultados do questionário IASFI mostraram que o GA apresentou maior grau de instrução e maior condição financeira comparado ao GS. Quanto às Atividades Básicas de Vida Diária (ABVD) e às Atividades Instrumentais de Vida Diária (AIVD), não houve diferença estatisticamente

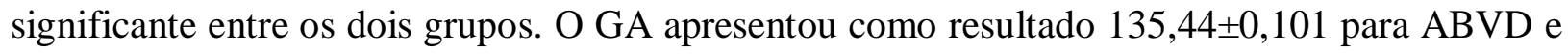

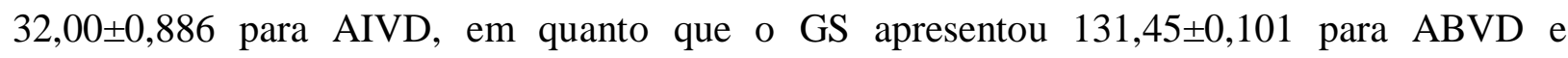
32,20 $\pm 0,886$ para AIVD. Não houve diferença estatisticamente significativa para a quantidade de quedas entre os dois grupos. As demais características sócio-demográficas estão descritas na Tabela1.

Tabela 1 - Características Sócio-Demográficas

\begin{tabular}{lcc}
\hline QUANTIDADE & ATIVO & SEDENTÁRIO \\
ESCOLARIDADE & 25 & 20 \\
$\quad$ Analfabeto & 0 & 1 \\
$1^{\circ} \mathrm{Grau}$ & 2 & 5 \\
$2^{\circ} \mathrm{Grau}$ & 19 & 8 \\
$3^{\circ} \mathrm{Grau}$ & 4 & 6 \\
ESTADO CIVIL & & \\
$\quad$ Solteiro & 1 & 4 \\
Casado & 6 & 9 \\
$\quad$ Divorciado & 4 & 2 \\
$\quad$ Viúvo & 14 & 5 \\
TRABALHO & 3 & 4 \\
Sim & 22 & 16 \\
$\quad$ Não & & \\
RENDA & 4 & 1 \\
Até 1 Salário Mínimo & 2 & 2 \\
1 a 2 Salários Mínimos & 2 & 1 \\
2 a 3 Salários Mínimos & 3 & 0 \\
3 a 4 Salários Mínimos & 1 &
\end{tabular}




\begin{tabular}{lcc}
+ de 5 Salários Mínimos & 13 & 7 \\
SAÚDE & 1 & 4 \\
Excelente & 15 & 8 \\
Boa & 9 & 8 \\
Regular & & \\
DOR & 20 & 18 \\
Sim & 5 & 2 \\
Não & & \\
QUEDA & 4 & 4 \\
Sim & 21 & 16 \\
Não & & \\
\hline
\end{tabular}

A pontuação obtida pela escala BERG mostrou uma média de 53,12 \pm 4,25 para o GA, contra 47,35 \pm 7,27 no GS apresentando uma diferença estatisticamente significante $(\mathrm{p}=0,002)$ entre os dois grupos estudados.

Dentre as variáveis avaliadas através do questionário SF-36, os domínios que apresentaram diferença estatisticamente significante $(\mathrm{p}<0,05)$ entre os dois grupos foram a Capacidade Funcional com maior valor para o grupo das idosas ativas e Estado geral de saúde mais evidente estatisticamente para o GS. Os dados obtidos pelo questionário SF-36 estão apresentados na Tabela 2.

Tabela 2 - Valores obtidos na comparação entre os grupos para cada domínio do questionário SF-36

\begin{tabular}{lccc}
\hline & ATIVO & SEDENTÁRIO & p \\
CAPACIDADE FUNCIONAL & $78,00 \pm 17,01$ & $59,75 \pm 23,75$ & $0,004^{*}$ \\
ASPECTOS FÍSICOS & $65,00 \pm 42,08$ & $55,00 \pm 45,59$ & 0,449 \\
DOR & $68,60 \pm 23,15$ & $63,20 \pm 21,30$ & 0,425 \\
ESTADO GERAL DE SAÚDE & $82,36 \pm 10,59$ & $91,10 \pm 09,62$ & $0,006^{*}$ \\
VITALIDADE & $77,60 \pm 18,88$ & $76,50 \pm 18,64$ & 0,846 \\
ASPECTOS SOCIAIS & $86,00 \pm 20,51$ & $66,87 \pm 45,21$ & 0,066 \\
ASPECTOS EMOCIONAIS & $82,66 \pm 37,41$ & $76,66 \pm 42,02$ & 0,615 \\
SAÚDE MENTAL & $81,44 \pm 13,41$ & $88,60 \pm 11,84$ & 0,068 \\
\hline
\end{tabular}

*p $<0,05$

\section{DISCUSSÃO}

Lima-Costa et al. ${ }^{(15)}$ referem em seu estudo que o Brasil se coloca como um dos países com maior nível de desigualdade social no mundo, fato esse evidenciado em nosso estudo frente à diferença encontrada entre as condições econômicas dos dois grupos avaliados. O GA 
apresentou maior nível de instrução e uma condição financeira muito mais favorável que no GS. As idosas que apresentaram maior renda tiveram uma auto-percepção da saúde mais positiva. Este achado é semelhante ao encontrado por Rocha e Freire, ${ }^{(5)}$ em que $32,1 \%$ das idosas fisicamente inativas possuia melhor percepção de saúde contra $67,9 \%$ das idosas fisicamente ativas, com significância de $\mathrm{p}=0,039$. A situação socioeconômica desempenha um papel fundamental na determinação das condições de saúde das pessoas, ${ }^{(16)}$ estando diretamente relacionada com uma autopercepção positiva da saúde. Por este motivo, as políticas de atenção à saúde do idoso visam conter ações voltadas à melhoria da condição de renda das pessoas. ${ }^{(5)}$

Não foram observadas diferenças estatisticamente significantes relacionadas às ABVD's e às AIVD's entre os dois grupos, corroborando com a evidência de Bocalini ${ }^{(17)}$ no qual refere que para a realização de ABVD's e AIVD’s é necessário o mínimo de aptidão física. A partir disso, reforça-se o fato de que a prática ou a não da atividade física, não interferem significativamente nas ABVD's e nas AIVD's. No entanto, existem divergências na literatura como no estudo de Franchi ${ }^{(18)}$ referindo que um estilo de vida fisicamente inativo pode ser causa primária da incapacidade para realizar AVD's.

A perda progressiva de massa muscular decorrente do envelhecimento causa redução do controle do centro de gravidade e consequenetemente no equilíbrio corporal. A prática de atividade física vem como uma modalidade terapêutica capaz de melhorar a mobilidade física e estabilidade postural que estão diretamente relacionadas às quedas em idosos. ${ }^{(11,19)}$ Esta afirmação se mostrou significativa no presente estudo no qual o GA apresentou um escore na escala de Berg significativamente melhor que o grupo GS, mesmo não se traduzindo em diferença significante para a ocorrência de quedas entre os dois grupos.

Com a aplicação do questionário de Berg, Resende ${ }^{(7)}$ encontrou uma melhora significativa do equilíbrio em idosas submetidas a um programa de hidroterapia com p=0,001, apresentando melhor resultado na diferença média após a $12^{\mathrm{a}}$ semana de tratamento, com o escore de 7,69 $\pm 3,77$. Outros estudos ${ }^{(6,21)}$ também confirmam esse achado reforçando o fato de que idosos praticantes de atividade física, apresentam um melhor escore na escala de Berg e dessa forma, uma melhor manutenção da postura, reduzindo o risco de quedas.

O envelhecimento representa, dentre outras perdas fisiológicas, o declínio da capacidade funcional e por outro lado, uma vida ativa pode manter a capacidade funcional nos idosos por mais tempo. ${ }^{(10,20)}$ As idosas ativas apresentaram maior capacidade funcional em nosso estudo. 
Nakagava ${ }^{(10)}$ encontrou em seu estudo resultado semelhante no qual as idosas praticantes de atividade física regular apresentaram um escore de capacidade funcional de 85,3 avaliado pelo questionário SF-36, valor este superior à média geral de todos os domínios, sugerindo uma melhor qualidade de vida proveniente dos benefícios da prática de atividade física.

Estado geral de saúde significa usufruir de uma condição de bem-estar que inclui o bom funcionamento do corpo, o vivenciar de uma sensação de bem-estar psicológico e principalmente uma boa qualidade nas relações que o indivíduo mantém com as outras pessoas e com o meio ambiente. ${ }^{(15)}$ Mota $^{(22)}$ comparou em seu estudo, a qualidade de vida em idosos ativos e não ativos através do questionário SF-36. Todos os domínios apresentaram significância a favor dos idosos ativos, dentre os quais o estado geral de saúde apresentou o valor 45,3 $\pm 18,3$ para os ativos e $66,1 \pm 15,4$ para os não ativos corroborando com o estudo de Toscano. ${ }^{\text {(23) }}$ Entretanto ambos estudos foram divergentes quando comparados ao presente estudo em que foi encontrado um melhor estado geral de saúde no GS.

Observou-se nos resultados referentes à qualidade de vida que a maior parte dos domínios não obteve resultados estatisticamente significantes entre os grupos. Este dado diverge da literatura dos estudos de $\operatorname{Mota}^{(22)}$ e Toscano ${ }^{(23)}$ que apresentaram resultados significativamente melhores para os idosos mais ativos em todos os domínios avaliados através do questionário SF-36. Possivelmente esta divergência foi encontrada devido ao fato dos indivíduos que não praticam atividades físicas propriamente ditas, não necessariamente são sedentários. Este conflito de conceitos também foi evidenciado por Palma ${ }^{(24)}$ referindo que este é um conceito "problemático" por ser uma expressão vulgar, comum e não científica, sendo um desafio para os pesquisadores determinar o que significa sedentarismo.

Os resultados obtidos através do presente estudo poderiam ser mais conclusivos com a adoção de um estudo de caráter longitudinal para evidenciar as causas e efeitos acompanhando os indivíduos avaliados, inclusão de uma maior amostra e com abordagem de uma amostra mais homogenia quanto aos dados sócios demográficos apresentados. Essas foram as limitações apresentadas pelo estudo e servem de contribuição para futuros trabalhos que venham a ser construídos.

\section{CONCLUSÃO}


No presente estudo, diante do melhor resultado de equilíbrio obtido pelos idosos praticantes de atividade física comparado aos idosos sedentários, sugere-se que a prática de atividade física em idosos influencia positivamente no equilíbrio corporal. Em relação à qualidade de vida foi observada diferença significativa em apenas dois domínios sugerindo que idosos fisicamente ativos se beneficiam de uma melhor capacidade funcional e os idosos sedentários, em dissonância com a literatura, apresentam melhor estado geral de saúde. Este último sugere que a literatura científica defina em consenso o conceito de sedentarismo para que novos estudos possam a longo prazo acompanhar a repercussão do sedentarismo na população idosa.

\section{REFERÊNCIAS}

1. Freitas EV de, Py L, Neri AL, Cançado FAX, Gorzoni ML, Rocha SM da. Tratado de geriatria e gerontologia. Rio de Janeiro: Guanabara Koogan; 2002.

2. Guedes RML. Motivação de idosos praticantes de atividades físicas. In: Guedes OC, organizador. Idoso, esporte e atividades físicas. João Pessoa: Idéia; 2001.

3. Costa MFL, Barreto SM, Giatti L, Uchôa E. Desigualdade social e saúde entre idosos brasileiros: um estudo baseado na Pesquisa Nacional por Amostra de Domicílios. Cad Saúde Pública. 2003; 19(3): 745-57.

4. Rocha SV, Freire MO. Nível de atividade física habitual e autopercepção do estado de saúde em idosas no município de Jequié - Bahia. Rev bras prom saúde. 2007; 20(3): 161-167.

5. Pedrinelli A, Garcez-Leme LE, Nobre, RSA. O efeito da atividade física no aparelho locomotor do idoso. Rev Bras Ortop. 2009; 44(2): 96-101.

6. Sanglard RCF, Pereira JS, Henriques GRP, Gonçalves GB. A influência do Isostretching nas alterações do equilíbrio em idosos. R bras ci. e mov. 2007; 15(2): 63-71.

7. Resende SM, Rassi CM, Viana FP. Efeitos da hidroterapia na recuperação do equilíbrio e prevenção de quedas em idosos. Rev Bras Fisioter. 2008; 12(1):57-63.

8. Grahn Kronhed AC, Möller C, Olsson B, Möller M. The effect of short-term balance training on community-dwelling older adults. J Aging Phys Activ. 2001; 9:19-31.

9. Bruni BM, Granado FB, Prado RA. Avaliação do equilíbrio postural em idosos praticantes de hidroterapia em grupo. O mundo da Saúde São Paulo. 2008; 32(1): 56-63.

10. Nakagava BKC, Rabelo RJ. Perfil da qualidade de vida de mulheres idosas praticantes de hidroginástica. MOVIMENTUM - Rev digit educ fís. 2007; 2(1): 1:14. 
11. Santos SR, Santos IBC, Fernandes MGM, Henriques MERM. Elderly quality of life in the community: application of the Flanagan's Scale. Rev Latino Am Enfermagem. 2002; 10(6): 757-64.

12. Fonseca FB, Rizzotto MLF. Construção de instrumento para avaliação sócio-funcional em idosos. Texto Contexto Enferm. 2008; 17(2): 365-73

13. Miyamoto ST, Lombardi Junior I, Berg KO, Ramos LR, Natour J. Brazilian version of the Berg balance scale. Braz J Med Biol Res. 2004; 37(9): 1411-1421.

14. Ciconelli RM, Ferraz MB, Santos W, Meinão I, Quaresma MR. Tradução para a língua portuguesa e validação do questionário genérico de avaliação de qualidade de vida SF-36 (Brasil SF-36). Rev bras Reumatol. 1999; 9(3): 143-50.

15. Lima-Costa MF, Matos DL, Camarano AA. Evolução das desigualdades sociais em saúde entre idosos e adultos brasileiros: um estudo baseado na Pesquisa Nacional por Amostra de Domicílios (PNAD 1998, 2003). Ciênc \& Saúde Coletiva. 2006; 11(4): 941-950.

16. Silva MAD, De Marchi R. Saúde e qualidade de vida no trabalho. São Paulo: Best Seller; 1997.

17. Bocalini DS, Santos RN, Miranda MLJ. Efeitos da pratica de dança de salão na aptidão funcional de mulheres idosas. R bras ci e mov. 2007; 15(3): 23-29.

18. Franchi KMB, Montenegro Junior RM. Atividade física: uma necessidade para a boa saúde na terceira idade. Rev bras prom Saúde. 2005; 18(3): 152-156.

19. Guimarães LHCT, Galdino DCA, Martins LFM, Vitorino DFM, Pereira KL, Carvalho EM. Comparação da propensão de quedas entre idosos que praticam atividade física e idosos sedentários, Rev neuroc. 2004; 12(2): 68-72.

20. Cosme RG, Okumai SS, Mochizuki L. A capacidade funcional de idosos fisicamente independentes praticantes de atividade física. R bras ci e mov. 2008; 16(1): 39-46.

21. Teixeira SC, Lemos LFC, Lopes LFD, Rossi AG, Mota CB. Equilíbrio corporal e exercícios físicos: uma investigação com mulheres idosas praticantes de diferentes modalidades. Acta Fisiatr. 2008; 15(3): 156-159.

22. Mota J, Ribeiro JL, Carvalho J, Matos MG. Atividade física e qualidade de vida associada à saúde de idosos participantes e não participantes em programas regulares de atividade física. Rev. bras. educ. fís. esp. 2006; 20(3): 219-25.

23. Toscano JJO, Oliveira ACC. Qualidade de vida em idosos com distintos níveis de atividade física. Rev bras med esporte. 2009; 15(3): 169-173.

24. Palma A. Exercício Físico e Saúde; Sedentarismo e Doença: Epidemia, Causalidade e Moralidade. Motriz. 2009; 15(1):185-191. 